CAHIERS DE

NARRATOLOGIE

\section{Cahiers de Narratologie}

Analyse et théorie narratives

17 | 2009

Stéréotype et narration littéraire

\title{
Une écriture entre les lignes de l'Histoire : fiction narrative et vérité historique dans Marie Stuart de Stefan Zweig
}

Frédérica Zephir

\section{OpenEdition}

Journals

Édition électronique

URL : http://journals.openedition.org/narratologie/6113

DOI : 10.4000/narratologie.6113

ISSN : 1765-307X

Éditeur

LIRCES

Référence électronique

Frédérica Zephir, «Une écriture entre les lignes de l'Histoire : fiction narrative et vérité historique dans Marie Stuart de Stefan Zweig », Cahiers de Narratologie [En ligne], 17 | 2009, mis en ligne le 07 juillet 2010, consulté le 15 novembre 2019. URL : http://journals.openedition.org/narratologie/6113

Ce document a été généré automatiquement le 15 novembre 2019.

\section{(c) (i) $\odot$}

Cahiers de Narratologie - Analyse et théorie narratives est mis à disposition selon les termes de la licence Creative Commons Attribution - Pas d'Utilisation Commerciale - Pas de Modification 4.0 International. 


\title{
Une écriture entre les lignes de l'Histoire : fiction narrative et vérité historique dans Marie Stuart de Stefan Zweig
}

\author{
Frédérica Zephir
}

1 Quand Stefan Zweig se propose d'aborder le personnage de Marie Stuart dans un ouvrage qu'il lui consacre en $1935^{1}$, il part du constat qu'aucune autre figure historique peut-être n'a, autant qu'elle, suscité de débats, de controverses chez les historiens, mais aussi de romans, de drames chez les écrivains et les poètes depuis plus de trois siècles. Pourtant, malgré la profusion d'études historiques et d'œuvres littéraires, l'inconnu, le mystère, qui s'attache à la personnalité et à la vie de la dernière reine d'Écosse, n'a jamais été dissipé.

2 La destinée tragique de cette femme, parce qu'elle se situe à un moment clé de l'histoire anglaise, a cristallisé toutes les passions nées du heurt de deux époques, du choc de deux visions du monde, de sorte qu'aucun des innombrables témoignages sur sa vie et aucun des développements et interprétations auxquels elle a donné lieu ne sont exempts du clivage dont sa vie porte les stigmates. Qu'ils soient catholiques ou protestants, anglais ou écossais, aucun commentateur, aucun historien, aucun romancier n'a pu écarter les voiles du mystère de Marie Stuart sans projeter sur sa vie l'ombre de sa propre religion ou de sa propre origine.

3 Partant de ce constat, Zweig imagine qu'un esprit neutre comme le sien, ni anglais, ni écossais, ni catholique, ni protestant, distant par le temps et la culture des préjugés qui ont troublé l'objectivité de ses prédécesseurs, peut aborder cette personnalité mû seulement par la ferveur de l'artiste et l'impartialité du psychologue. Ainsi élabore-t-il une œuvre dont la singularité, conjuguée à la richesse de la peinture du personnage, ne peut manquer de soulever quelques questions.

4 Si l'on considère en effet le portrait de l'héroïne et qu'on le compare, par exemple, à celui brossé par Walter Scott dans l'Abbé, on peut se demander qu'elle est la part de 
l'objectivité, et si Zweig dans son "essai ", n'a pas tout autant transfiguré le modèle historique que le romancier écossais; si sa neutralité, garantie par son impartialité envers les causes idéologiques et politiques antagonistes de l'époque, n'est pas subvertie par sa sensibilité même d'artiste.

Nous nous proposons donc d'établir, qu'en dépit du projet attesté de l'auteur, sa reine d'Écosse est, notamment dans sa psychologie, un personnage fortement fictionnel et que l'œuvre dans son ensemble peut être rattachée au statut générique du roman historique.

Dans cette perspective, il semble tout d'abord nécessaire d'analyser la façon dont Zweig aborde le personnage de Marie Stuart, c'est-à-dire d'observer comment, influencé en cela par une longue tradition littéraire, il la conçoit d'emblée comme une figure non pas purement historique, mais située entre l'Histoire et la légende. Car si, comme nous tenterons de le montrer ensuite, Marie Stuart apparaît bien dans cet "essai » comme une véritable héroïne zweigienne illustrant les deux grands motifs que l'écrivain déroule tout au long de son œuvre - celui du déclin du monde des valeurs humanistes et celui de la passion - c'est que les lacunes de l'analyse historique jointes au mythe poétique forgé autour de l'héritière d'Écosse lui ouvrent la voie d'une interprétation personnelle de la vie hors norme et des actes singuliers de cette souveraine. En investissant ainsi le personnage historique de sa propre thématique tout en conservant - et c'est ce qui fait la richesse et la complexité de son récit - une parfaite rigueur documentaire dans la présentation des événements, Stefan Zweig élabore donc une œuvre des plus originales dont nous nous efforcerons enfin de définir, entre fiction narrative et vérité historique, le statut particulier en montrant comment l'artiste intègre et dépasse les contradictions génériques par une saisie empathique de son personnage et un art achevé de la biographie.

\section{Marie Stuart, une figure entre Histoire et légende}

7 Mais avant d'aborder, entre Histoire et légende, l'image de Marie Stuart, rappelons brièvement les étapes principales de son existence mouvementée. Reine d'Écosse cinq jours après sa naissance en 1542, elle devint Reine de France par son mariage avec le Dauphin François, fils d'Henri II et de Catherine de Médicis. Veuve en 1560, elle retourna en Écosse où elle s'unit à son cousin Henry Darnley. Un an et demi plus tard celui-ci fut assassiné par le comte de Bothwell que la reine épousa en troisièmes noces. Rivale de sa cousine Elisabeth $1^{\text {ère }}$, celle-ci la retint captive en Angleterre pendant près de vingt ans où elle fut finalement condamnée à mort pour conspiration et exécutée le 8 février 1587.

\section{Sous le signe de Sophocle et de Shakespeare :}

\section{Marie souveraine de la terre tragique des thanes}

8 Évoquer Marie Stuart, c'est d'abord évoquer son siècle, ce XVI ${ }^{\mathrm{e}}$ siècle qui lui ressemble tant, illuminé à son commencement par le rayonnement de la Renaissance et s'abîmant peu à peu dans le fanatisme et les horreurs des guerres de religion. Ce siècle qui voit successivement briller au firmament des lettres le lyrisme de la Pléiade, l'humanisme de Montaigne et, avec Shakespeare, renaître et s'épanouir l'art de la tragédie. Sans 
doute cette analogie entre l'évolution littéraire du siècle et la destinée de son personnage n'a-t-elle pas échappé à Zweig pour qui Marie Stuart, chantée dans son adolescence par Ronsard et Du Bellay, s'inscrit d'abord dans l'histoire comme une personnalité hautement tragique héritière des héroïnes grecques et parente des plus sombres personnages shakespeariens. Dès le début de son essai en effet Zweig insiste sur cette proximité en croyant voir se manifester l'antique fatum dans la lignée même de Marie, fille de Jacques V d'Écosse et de Marie de Guise, et en comparant d'emblée la dynastie des Stuart aux Atrides :

Mais c'est un héritage doublement fatal que d'être reine d'Écosse et une Stuart en même temps [...] Aucun de ces nouveaux Atrides n'a pu atteindre le sommet de la vie $^{2}$.

De même, lorsqu'il se penche sur le rôle tenu par Marie Stuart dans l'assassinat de Lord Darnley, c'est encore Clytemnestre qu'il évoque, se demandant si, à l'instar de la reine mythique, Marie Stuart, poussée par sa folle passion pour Bothwell, n'a pas traîtreusement attiré son mari dans le piège tendu par celui-ci en vue de l'épouser et de s'emparer ainsi de la couronne.

10 Mais si Marie Stuart évoque bien pour Zweig la figure légendaire de la reine d'Argos, elle s'apparente plus encore pour lui aux héros shakespeariens, les moments les plus dramatiques de sa vie lui apparaissant comme de véritables actes de tragédie. Car reviennent en effet de façon récurrente tout au long de l'œuvre les comparaisons entre les événements réels de la vie de la reine et les pièces de l'illustre dramaturge. Ainsi, lorsque Marie Stuart, poussée à la fois par son amour pour Bothwell, la haine de son mari et son désir de se venger de lui, se retrouve entraînée dans la voie du crime, c'est tout naturellement que surgit le personnage de Lady Macbeth « endormant avec de douces et caressantes paroles le roi Duncan que Macbeth assassinera pendant son sommeil $^{3}$ ». De la même façon, Zweig évoquera Richard III et Hamlet à propos du mariage de Marie et de Bothwell quelques semaines à peine après le meurtre de Darnley.

11 Au reste, la référence à la tragédie shakespearienne est perceptible au-delà même du personnage de Marie Stuart dans l'évocation de l'atmosphère générale du pays qu'elle gouverne. Car si Zweig perçoit le tragique à l'œuvre dans la longue lignée des Stuart, il l'appréhende aussi dans le contexte historique du royaume d'Écosse "pays sévère et tragique ${ }^{4}$ » selon ses propres termes, sans cesse déchiré par les luttes intestines entre les différents clans; car c'est bien sur la farouche terre des thanes sanglants, cousins de Macbeth et de Macduff, que règne la dernière reine d'Écosse.

\section{Hécate chez Lady Macbeth}

12 Dépositaire d'un héritage marqué par la violence du destin, souveraine d'une terre meurtrie où le malheur a creusé ses sillons, Marie Stuart, chez Zweig, apparaît en outre elle-même comme une figure de la fatalité entraînant dans son sillage funeste tous les hommes qui gravitent autour d'elle, fascinés par sa personnalité et captivés par l'attrait du pouvoir qu'elle incarne. Égrenant, du troubadour Chastelard au Duc de Norfolk en passant par le secrétaire Riccio, le gentilhomme Babington et bien sûr son mari Lord Darnley, la longue liste de tous ceux qui périrent en la servant ou en l'aimant, Zweig façonne, au fil de sa biographie, un personnage au charme fatal, une sorte d'Hécate, incarnation maléfique de la lune, symboliquement représentée par la "reine noire » des dernières années de captivité ; « Une fois de plus, écrit-il, le sang a coulé pour cette 
femme à qui a été donné le pouvoir fatal d'entraîner sans cesse de nouvelles victimes dans son orbe tragique ${ }^{5}$ "

Figure atridienne ou shakespearienne, être fatal dont le destin attire sinistrement à lui d'autres destins, Marie Stuart apparaît donc bien, dans la vision zweigienne, comme un personnage fondamentalement tragique dont la vie semble irrémédiablement frappée par un sort impitoyable. D'autant qu'aux prises avec de graves problèmes politiques que sa jeunesse et son inexpérience ne lui permettaient pas de maîtriser, elle se trouva opposée, en un duel sans merci, à sa puissante et habile cousine Elisabeth pendant près de vingt années.

\section{Le duel des Dames : Marie Stuart contre Elisabeth d'Angleterre}

\section{Le contentieux entre Marie et Elisabeth}

$14 \mathrm{Si}$, comme nous avons tenté de le montrer, Marie Stuart se présente, dans la vision zweigienne, comme un personnage fondamentalement tragique, la tragédie qu'est sa vie ne prend sa pleine dimension que par sa confrontation avec l'autre protagoniste, celui qui, par son existence même, est l'agent de la fatalité et va frapper le coup décisif qui érigera l'existence de la reine d'Écosse en destin. En effet, dès le retour de Marie Stuart dans son pays en 1561, le contentieux qui l'oppose à Elisabeth, reine d'Angleterre depuis 1558 , se réactive et scelle en un conflit implacable la rivalité entre les deux souveraines. Car si les ambassadeurs ont bien conclu à Édimbourg un traité par lequel Marie Stuart s'engage à reconnaître "for all times coming ${ }^{6}$ » la légitimité d'Elisabeth sur le trône d'Angleterre, jamais la jeune reine n'accepta de le ratifier, entérinant ainsi de son plein gré, par honneur et orgueil dynastique, une erreur politique commise, au temps de son mariage avec le Dauphin de France, sous l'influence de sa parentèle.

15 Car Elisabeth ne pouvait évidemment laisser peser une telle ambiguïté sur ses droits, tolérer la menace que constituaient pour elle les revendications de sa cousine sur la couronne d'Angleterre. Mais Marie Stuart, dont la filiation et la complexité du testament d'Henri VIII autorisaient les ambitions, ne voulait à aucun prix renoncer à ses droits à la succession d'Angleterre et exigeait d'Elisabeth qu'elle la reconnût comme son héritière officielle, ce à quoi celle-ci se refusait absolument en raison notamment du catholicisme de la reine d'Écosse. Ainsi, aucune des deux reines ne voulant céder à l'autre, les composantes de la tragédie étaient bien réunies puisque le nœud gordien ne pouvait plus désormais être tranché que par la disparition d'une des deux souveraines.

\section{La question religieuse}

D'autant qu'à ce contentieux dynastique s'ajoutaient encore les tensions religieuses qui, secouant l'Europe depuis déjà plusieurs années, commençaient à l'époque à diviser l'Écosse. Entrainés par les prêches enflammés du pasteur John Knox, les Protestants, soutenus par l'Angleterre, s'élevaient en effet contre l'accession au trône d'une reine catholique. Et, quoique Marie Stuart peu encline aux excès du fanatisme religieux, offrît des garanties et des gages de tolérance évidents, elle ne put désarmer la haine antipapiste de l'inflexible pasteur. Car, dans cette confrontation, la jeune souveraine se voyait soumise à des enjeux qui la dépassaient et sur lesquels sa volonté n'avait aucune prise, son destin étant tributaire des forces supérieures de l'Histoire comme le souligne Zweig dans ces lignes : « Par un hasard étrange le conflit entre Luther, Calvin et Rome a 
été transporté dans sa vie d'une façon dramatique, la lutte entre Marie Stuart et Elisabeth, entre l'Angleterre et l'Écosse [...] c'est aussi la lutte entre l'Angleterre et l'Espagne, entre la Réforme et la Contre-Réforme ${ }^{7}$. ».

Incarnant ainsi dramatiquement dans sa destinée personnelle les bouleversements d'une époque marquée par la rupture entre une société encore largement féodale et un monde fondé sur des valeurs nouvelles, la Marie Stuart de Zweig s'inscrit bien au cœur même de l'Histoire, tout en conservant, par son aspect d'hérö̈ne tragique, l'aura mythique qui nimbe sa personnalité.

18 Mais en se penchant sur les contradictions et les tensions internes révélées par les actes les plus énigmatiques de la souveraine, Zweig dévoile encore d'autres aspects du personnage. Traçant d'abord, à la faveur de la comparaison avec Elisabeth, le portait d'une femme sublime, il met ensuite en évidence la dimension structurante des valeurs et de la culture humaniste dans sa formation qui en fait, à ses yeux, la dernière héroïne d'un monde en train de disparaître. Enfin, en faisant ressortir le caractère destructeur de sa passion amoureuse, il confère à sa reine d'Écosse une dimension nouvelle et très personnelle, octroyant ainsi au personnage une épaisseur que l'analyse historique seule ne saurait restituer.

\section{Un personnage Zweigien}

\section{Portrait de Marie Stuart}

19 Bien que Zweig nomme essai et non biographie son œuvre consacrée à Marie Stuart, il y compose néanmoins une des plus subtiles peintures psychologiques qui ait été donnée de la dernière reine d'Écosse. Saisissant ce qu'il y d'irrationnel et de contradictoire dans sa personnalité, il élabore le portait d'une femme à la fois douce, spontanée, insouciante et pleine d'alacrité mais aussi d'un être passionné et impulsif, possédé en outre par un orgueil farouche dès qu'il s'agit de ses prérogatives royales

\section{De la reine au berceau à la reine déchue : dévoilement d'une femme sublime}

Née reine, Marie Stuart ne peut en effet accepter le moindre manquement à ses droits régaliens. Dès que sa place n'est plus la première ou dès que sa dignité de reine lui paraît outragée, cette femme plutôt douce et insouciante selon Zweig devient "subitement dure, audacieuse, arrogante dès que son honneur est en jeu ${ }^{8}$. Et c'est d'ailleurs cette susceptibilité qui explique en grande partie le contentieux qui l'opposa à Elisabeth, Marie Stuart ne voulant à aucun prix renoncer à ses droits sur la couronne d'Angleterre. Outre cet inflexible orgueil dynastique au service duquel elle peut de surcroît déployer un courage qui, confinant souvent à l'héroïsme, confère au personnage une dimension sublime, la reine d'Écosse révèle un caractère fortement contrasté.

21 En effet, de même qu'elle dissimule sous un corps frêle et gracieux une étonnante résistance physique qui en fait notamment une cavalière infatigable, de même sous des dehors de douceur, des manières accortes et un comportement assuré, elle recèle « un prodigieux potentiel de passion ${ }^{9}{ }^{\prime}$. Dotée d'un caractère ardent et impulsif, elle n'agit jamais de façon "prudente et réfléchie ${ }^{10}$ ", mais plutôt dans des moments d'exaltation qui contrastent avec son naturel d'ordinaire indolent. Ainsi par cette douceur, cette 
grâce, cette nature lumineuse symbolisée par la "reine blanche » (de Fontainebleau) immortalisée par Clouet, Marie Stuart se rattache bien à la Renaissance, mais par l'autre versant de sa personnalité, par son caractère ardent et sa passion qui l'emporte et lui ôte parfois la maîtrise d'elle-même, elle est déjà, pour Zweig, un personnage romantique que symbolise alors la « reine noire » des dernières années. De sorte que l'on pourrait remarquer qu'ainsi définie par cette opposition fondamentale, la Marie Stuart de Zweig paraît dès lors condenser en elle deux types d'héroïnes romanesques l'une de nature claire, l'autre de nature sombre - fréquentes, selon Northrop Frye, dans le roman du XIX ${ }^{e}$ siècle, et notamment dans le roman Scottien.

\section{Une personnalité romantique}

Quoi qu'il en soit, il reste qu'une des caractéristiques essentielles du portrait de Marie Stuart réside bien dans cet aspect romantique du personnage allié à la dimension tragique que nous avons déjà évoquée. Car si Zweig situe la souveraine d'Écosse dans la lignée des grandes hérö̈nes grecques ou shakespeariennes, il la perçoit aussi comme une personnalité foncièrement romantique. Cette épithète, dont la récurrence dans le texte atteste l'importance, recouvre dans la vision de Zweig plusieurs significations. Car romantique, Marie Stuart l'est d'abord au sens où Madame de Staël entend ce terme, c'est-à-dire par ce qu'il y a en elle de chevaleresque, par sa courtoisie, son énergie, son courage et son héroïsme c'est-à-dire par ce qu'elle incarne encore des valeurs du Moyen Age, éclairant : « d'un dernier reflet de la chevalerie et des troubadours ce pays froid et brumeux que vient encore obscurcir l'ombre de la Réforme ${ }^{11}$ ».

Mais elle l'est aussi, au sens plus traditionnel, par sa sensibilité, son idéalisme et surtout, par la violence de ses sentiments, qui en font selon Schiller "un être d'instincts naturels (dont) la destinée est d'éprouver et de déchaîner des passions violentes ${ }^{12}$ ». Et sans doute est-ce afin de conforter cet aspect et d'expliquer par là la folle passion que Marie éprouva pour lui, que Zweig appréhende Bothwell lui-même comme une figure hautement romantique: «... il a gardé [...] l'amour effréné de l'aventure de ces "hors-la-loi ", de ces corsaires romantiques, célébrés par Byron ${ }^{13}$ ". Mais c'est dans le face à face avec Elisabeth, dans l'irréductible opposition des deux reines, analysés par Zweig avec beaucoup de pénétration- mais non sans parti pris- que s'affirme cette dimension, reprise et exaltée par la plupart des auteurs que l'héritière des Stuart inspira, Walter Scott et Schiller notamment. Car si Zweig est fasciné par la personnalité de Marie Stuart, il ne l'est pas moins par l'étonnant hasard historique qui préside à sa confrontation avec l'autre personnage d'exception qu'est Elisabeth $1^{\text {ère }}$, en laquelle il voit une sorte de double inversé de la reine d'Écosse, de sorte que c'est à partir de leur opposition psychologique et en se livrant à une véritable peinture comparative des deux souveraines qu'il élabore le portrait de son héroïne.

Il est en effet frappant de constater comment, dans la vision zweigienne, chaque point du caractère de Marie Stuart trouve son exacte antithèse dans celui d'Elisabeth ; de voir la spontanéité, l'audace de l'une s'opposer à la prudence et à l'esprit calculateur de l'autre, la générosité de l'héritière des Stuart à la parcimonie de la fille d'Henri VIII, sa sincérité et son incapacité à feindre trancher avec le tempérament ambigu voire hypocrite de celle que Zweig qualifie à plusieurs reprises de "comédienne née "; l'ardeur à vivre et à jouir dans le présent de Marie Stuart contraster avec le sens de l'Histoire développé par la reine d'Angleterre, et enfin l'idéalisme, le conservatisme de la dernière souveraine d'Écosse se heurter au réalisme politique de celle qui ouvre à son 
pays la voie du leadership mondial pour plusieurs siècles. Il n'est pas jusqu'au style vestimentaire qui ne s'oppose chez ces deux femmes, le charme et la noblesse innée de Marie Stuart contrastant avec le faste guindé des tenues sous lesquelles Elisabeth dissimulait, selon Zweig, sa fragilité intérieure.

Définies ainsi par leur opposition fondamentale, chacune des deux reines s'impose alors à la postérité dans un registre conforme à sa personnalité et à sa trajectoire singulière ; Elisabeth avec son esprit politique et son réalisme l'emportant dans le domaine de l'Histoire, Marie Stuart, dont la comparaison avec sa cousine assoit définitivement la dimension romantique, triomphant dans celui de l'esprit et de la légende.

\section{Une étoile au firmament de l'Humanisme}

\section{Marie Stuart incarnation de l'idéal de la Renaissance}

Parmi les traits essentiels de Marie Stuart que la vision zweigienne s'attache à développer, la dimension humaniste se révèle comme l'une des plus fondamentales. Pour Zweig en effet la vie de la dernière reine d'Écosse, avec ses lumières et surtout ses ombres, ne peut être appréhendée de façon pertinente sans tenir compte de l'influence profonde exercée sur elle par l'esprit de la Renaissance. Élevée, à partir de l'âge de cinq ans et demi, à la cour des Valois où triomphait alors, avec l'influence italienne, le culte des arts et des lettres, Marie Stuart a baigné dès ses plus jeunes années, dans l'ambiance raffinée d'une des cours les plus brillantes d'Europe. Durant cette période, recevant une éducation conforme à l'idéal de ce temps, elle apprit le grec, le latin, l'italien mais aussi la musique et la danse qu'elle pratiquait, dit-on, avec un charme et une grâce inégalés. Dans l'ombre de Ronsard et de Du Bellay, elle s'initia à la poésie qui restera pour elle, même aux plus sombres heures de sa vie, la forme d'expression privilégiée. Suivant la cour itinérante d'Henri II du Louvre à Amboise, de Blois à St Germain en Lay, participant aux fêtes splendides qui ponctuaient les grands événements et dont son mariage avec le Dauphin fournit une magnifique illustration, évoluant dans l'atmosphère de culture et de raffinement intellectuel auréolant cette cour somptueuse, Marie Stuart s'imprégna des plus hautes valeurs de la Renaissance au point d'en devenir aux yeux de son biographe l'incarnation la plus emblématique, "Sa seule jeunesse, écrit-il, symbolise d'une façon vraiment typique l'idéal de l'époque, où se trouvent réunis le courage et la légèreté, la force et la douceur sous une forme romanesque et audacieuse ${ }^{14} »$.

Indéfectiblement marquée par la richesse spirituelle, l'élégance et la douceur des mœurs de la cour des Valois et constatant bien vite "qu'elle est passée d'une civilisation riche, prodigue et jouisseuse dans un monde étroit, sombre et tragique ${ }^{15}$ ", Marie Stuart n'a de cesse de recréer, dès son retour en Écosse, une vie de cour lui rappelant les belles heures passées jadis en France, s'entourant de musiciens français et italiens, cultivant la poésie, organisant dans son château d'Holyrood des soirées où l'on chante des madrigaux et où l'on lit des vers, elle tente dans cette cour en miniature de ressusciter l'esprit de la Renaissance.

Et c'est dans cette intention qu'elle accorde de plus en plus de place et de faveurs à David Riccio, cet italien doué d'une belle voix qu'elle avait d'abord engagé comme chantre et qui deviendra ensuite son secrétaire et l'homme de confiance auquel elle confiera de plus en plus de pouvoir, suscitant ainsi l'hostilité des Lords et de son frère 
le Comte de Morray contre cet étranger catholique, dont l'assassinat frappera le premier coup de sa tragique existence.

Par ailleurs, il paraît indubitable que cet esprit de la Renaissance dont Marie Stuart était si profondément pénétrée ne soit l'un des facteurs déterminants de la lutte sans merci que le pasteur John Knox lui livra dès son arrivée sur le sol écossais. Puritain inflexible, disciple de Calvin, le chef de l'Église Réformée d'Édimbourg s'opposa en effet immédiatement à cette souveraine catholique de dix-huit ans arrivant de France et qui prétendait faire souffler sur sa nouvelle cour l'esprit brillant de cette Renaissance qu'elle incarnait si bien. Galvanisant les foules par ses sermons enflammés, John Knox dénonçait avec un tel fanatisme aussi bien le catholicisme de la reine (qu'elle ne pratiquait pourtant qu'en privé) que le style de vie, à ses yeux diabolique, au château d'Holyrood qu'aucun accord, aucun compromis ne pouvait s'établir entre deux personnalités si antagonistes :

Rien ne pouvait être plus contraire à sa manière d'envisager l'existence et d'en jouir, à ses penchants artistiques que cette froide austérité, cette attitude hostile envers la vie [...] cette haine de la joie qui s'affirmait dans la doctrine genevoise ; rien de plus insupportable pour elle que cette rigidité hautaine qui défend le rire et condamne le beau comme un crime, qui veut détruire tout ce qui lui est cher, les formes joyeuses des mœurs, la musique, la poésie et la danse ${ }^{16}$.

\section{L'humanisme de Zweig}

En évoquant ce duel farouche entre la reine et John Knox et, au-delà de celui-ci, l'opposition irréductible entre l'esprit humaniste représenté par Marie Stuart et ce qu'il nomme le « caractère talmudique ${ }^{17}$ » de l'Église Reformée d'Écosse, Zweig révèle le lien qui le relie à son hérö̈ne, lien qui, d'une part, permet de comprendre l'empathie qu'il ressent envers cette personnalité historique et qui, d'autre part, explique comment celle-ci, en illustrant un des thèmes privilégiés de l'auteur, devient elle-même un véritable personnage zweigien.

En effet ce qui séduit Zweig chez Marie Stuart c'est d'abord cet aspect de sa personnalité façonnée par les idéaux de la Renaissance. Ce goût raffiné, cet amour des arts et de la culture, cette primauté accordée à l'esprit ; cet esprit de tolérance enfin qui rejette tous les fanatismes et rêve de créer un univers où s'harmonisent les contraires, " force et beauté, bravoure et légèreté ${ }^{18}$ " sous la souveraineté de l'esprit. Or ce sont là des valeurs dont Zweig lui-même se réclame; valeurs qui prévalaient dans les cercles viennois de sa jeunesse et présidaient à l'effervescence intellectuelle de la Mittel Europa, deux décennies avant le premier conflit mondial. Car ce «jeune bourgeois voué au spirituel et au culte de l'art ${ }^{19}$ ", comme le définit Serge Niemetz dans sa biographie, reste indéfectiblement attaché à cette culture humaniste européenne dont le centre intellectuel était Vienne et qui «se posait en héritière de tout le passé prestigieux de l'humanité et en premier lieu de

l'Antiquité ${ }^{20} »$. Esprit insatiable, assoiffé d'idéal, c'est en effet vers l'art qu'il se tourne avec enthousiasme dès son adolescence, demandant à la musique, au théâtre,

à la littérature et à la poésie de le libérer de l'étroitesse et des pesanteurs du milieu bourgeois auquel il appartient. Ainsi est-ce sur «le sens du beau », "la passion des arts » et «les douces joies de l'esprit ${ }^{21}$ » qu'elle cultivait que se fonde la relation singulière unissant l'auteur à son personnage, Zweig reconnaissant en Marie Stuart un modèle accompli de personnalité humaniste, laquelle constitue à ses yeux le type humain le plus parfait que la civilisation occidentale ait engendré. 

situation historique, sa vie lui semblant en effet marquer la transition non seulement entre deux époques mais, plus profondément, entre deux conceptions du monde, de sorte que la dernière reine d'Écosse lui apparaît à plus d'un titre comme une véritable héroïne de "fin de monde ». Ainsi voit-il dans son départ de France l'événement qui annonce symboliquement le déclin de la Renaissance, les valeurs humanistes étant à partir de ce moment balayées par le déchaînement du fanatisme et des haines religieuses qui aboutiront onze ans plus tard, à la nuit de la Saint- Barthélemy :

Il n'y a que les poètes qui ressentent une tristesse sincère, un chagrin réel en voyant partir Marie Stuart [...] Ils savent que les muses quittent la France en même temps que cette jeune femme qui voulait y créer une cour de gaieté et de beauté : pour eux, comme pour tous, de sombres années approchent : années de luttes politiques et religieuses avec la Saint-Barthélemy et le règne du fanatisme. Finis l'éclat chevaleresque et romantique, le triomphe du beau et des arts. La Pléiade pâlira bientôt dans le ciel obscurci par la guerre. Les douces joies de l'esprit s'en vont avec Marie Stuart ${ }^{22}$.

\section{Une héroïne de « Fin de monde »}

Or c'est de semblable façon que la richesse intellectuelle et artistique caractérisant la Vienne Fin de siècle sera elle aussi anéantie, d'abord par le carnage de la Première Guerre Mondiale, puis par la barbarie du régime nazi. De sorte que Zweig, qui vécut la première comme l'écroulement d'un monde dans lequel il voyait l'âge d'or de la culture européenne, établit une relation d'analogie entre sa propre époque et celle de son personnage, analogie qui renforce la fascination qu'il éprouve à son égard.

Au reste, si Marie Stuart apparaît à Zweig comme la dernière étoile au firmament de l'humanisme, c'est sa vision politique tout entière qui lui semble se rattacher à un monde finissant. Et c'est une nouvelle fois dans son face à face avec Elisabeth d'Angleterre que, selon lui, se révèle avec une pathétique évidence cette fatale mais inévitable appréhension des événements de l'Histoire. Car Marie Stuart, éduquée à la cour des Valois, ancienne Reine de France, catholique apparentée aux Guise de surcroît, apparaît prisonnière d'une conception du monde qui, dans la seconde moitié du XVIe siècle, se trouvait remise en question par les bouleversements du monde consécutifs à l'extension de l'espace géographique résultant des grandes découvertes et, surtout, à l'ébranlement du catholicisme par la Réforme. Mais la reine d'Écosse, à la différence de sa cousine Elisabeth, ne semble pas avoir eu l'intuition des changements de mentalité engendrés par ces transformations, son histoire et sa formation ne lui permettant, au reste, ni de renoncer à sa religion ni d'imaginer une évolution du pouvoir monarchique. Or, s'il y a une indéniable grandeur dans la fidélité de Marie Stuart à la religion

de ses pères, il n'y a pas moins, selon Zweig, un aspect par trop conservateur pour l'époque dans cette conception rigide du pouvoir, même si son caractère inéluctable lui confère une dimension sublime : «Marie Stuart, [...] combat et tombe pour une cause ancienne, arriérée, en vrai paladin. Esprit rétrograde, elle obéit à la volonté de l'Histoire en s'alliant aux puissances qui ont déjà dépassé leur zénith, l'Espagne et la Papauté [...] La reine d'Écosse et la reine d'Angleterre incarnaient deux conceptions différentes du monde, Marie Stuart le monde expirant [...] Elisabeth le monde futur, les temps nouveaux. [...] La vie d'Elisabeth personnifie l'énergie d'une nation qui veut conquérir l'univers, la fin de Marie Stuart, c'est la mort héroïque et sublime d'une époque ${ }^{23} »$. 

même, si l'on suit Zweig, par sa religion, Marie Stuart l'est encore par sa tragique fin. Premier monarque de l'histoire européenne à être jugé et exécuté, sa mort marque en effet, plus que l'achèvement d'une époque, l'ébranlement de l'ordre même du monde, la souveraineté de droit divin, qui constituait jusqu'alors le fondement de la société, étant ainsi définitivement remise en question. Car l'exécution de Marie Stuart donnait à voir au peuple - à tous les peuples - que le roi était un homme comme les autres, responsable de ses actes et devant en rendre compte à d'autres hommes, lui qui n'était jusque-là comptable qu'envers Dieu. Cette désacralisation de la personne royale représentait un renversement radical des valeurs dont les conséquences se feraient sentir bien au-delà de la mort de Marie Stuart, puisque la hache qui brisait sa vie frappait le premier coup du glas qui retentirait pour son propre petit fils Charles $1^{\text {er }}$ d'Angleterre et résonnerait encore, plus de deux cents ans plus tard, pour Louis XVI et Marie-Antoinette. Et sans doute Elisabeth, qui hésita si longtemps avant de signer l'ordre fatal, pressentait-elle l'impact et les répercussions d'une telle décision sur l'ordre et l'équilibre des sociétés monarchiques.

Faisant briller pour la dernière fois la lumière de la Renaissance, héroïne d'un monde qui meurt, Marie Stuart apparaît bien ainsi comme une hérö̈ne zwegienne, illustrant parfaitement l'idée du déclin d'une époque, de la fin des valeurs humanistes qui faisaient la grandeur de ce "Monde d'Hier»-selon le titre de son autobiographie dans lequel Zweig voyait l'apogée de la civilisation européenne. Mais c'est aussi en développant de façon remarquable le motif de la passion - autre thème récurrent du romancier viennois - que le personnage de la Reine d'Écosse se révèle comme une figure exemplaire de son œuvre.

\section{La passion selon Zweig}

\section{La crise passionnelle}

En effet, captivé comme beaucoup d'autres, par l'aura de mystère qui enveloppe la vie sentimentale de Marie Stuart, Zweig s'efforce dans son essai d'expliquer le caractère énigmatique de la relation qui, la liant au Comte de Bothwell, a précipité sa destinée. Analysant cette liaison, Zweig examine en premier lieu l'amour que Marie éprouva tout d'abord pour Lord Darnley son second mari, car en lui résident, à son avis, les mécanismes profonds de ce qui se présente alors sous la forme d'une véritable crise. En effet Marie Stuart, la jeune veuve de François II, garçon chétif mort à seize ans auquel elle avait été unie par ce qu'il nomme « un mariage d'enfants ${ }^{24}$ », n'a aucune expérience véritable de l'amour lorsqu'elle s'éprend de son cousin Henry Darnley. Immédiatement éblouie par ce séduisant adolescent au physique avantageux, elle éprouve à son égard un sentiment tellement fort que, subjuguant sa raison et réduisant jusqu'à ses ambitions dynastiques, il la contraint contre tous les avis, à épouser ce prétendant si peu digne du titre de roi qu'elle s'empresse de lui octroyer; de sorte qu'en l'espace de quelques semaines seulement sa vie bascule, bouleversée par un véritable séisme affectif qui annihile sa volonté, hypothèque son avenir et celui de l'Écosse entière conduite par là aux portes de la guerre. La soudaineté de cet ébranlement intérieur, le caractère irréversible de ce qui apparaît comme une rupture dans la trame de l'existence ordinaire du personnage étant clairement attestés dans la narration : 
Hier, Marie Stuart était encore une politicienne, aujourd'hui, elle n'est plus rien, qu'une femme, qu'une amante. Être reconnue héritière du trône d'Angleterre était il y a quelques semaines le rêve de sa vie, aujourd'hui cette royale ambition disparaît devant le désir plus modeste mais plus impérieux de la femme : avoir, posséder ce jeune et joli garçon qu'est Darnley ${ }^{25}$.

C'est dans ce premier bouleversement affectif, lié à l'éveil de la sensualité, que résident les prémices de la grande passion qui submerge ensuite Marie Stuart comme d'autres personnalités féminines de l'œuvre dont l'héroïne de la nouvelle intitulée L'amour d'Erika Ewald offre, par exemple, une parfaite illustration :

Tout allait prendre une autre tournure que dans les rêves d'Erika. L'amour réapparut dans sa vie, mais il avait changé; il ne s'approchait plus d'un pas tranquille, comme une jeune fille, chargé de tendres présents et de bénédictions, mais comme un orage printanier comme une femme enflammée de désir, aux lèvres ardentes, et qui porte dans sa chevelure sombre la rose écarlate de la passion ${ }^{26}$.

Cette passion, dont la métaphore de l'orage exprime ici la violence, va donc faire irruption dans la vie de Marie Stuart et en précipiter le cours puisque c'est l'assassinat de Darnley et son remariage avec Bothwell qui scellent son tragique destin. Mais c'est d'abord sur la personnalité profonde de la reine que se font sentir les effets dévastateurs de son aveuglante passion. Car c'est transformée et complètement brisée que Marie Stuart sort des deux années que durent ses relations avec Bothwell. Transformée d'abord parce que cette femme altière et indomptable se soumet désormais aux volontés de cet homme brutal et ambitieux jusqu'à accepter l'idée d'éliminer son mari, mais aussi parce que cette figure confiante, insouciante et lumineuse semble soudain habitée par une force sombre et destructrice. C'est que, chez Zweig, la passion ravage tout sur son passage, semblable à une lave ardente trop longtemps contenue, elle consume l'être qu'elle emporte dans son déferlement laissant derrière elle un sujet intérieurement dévasté, une personnalité psychiquement anéantie, une vie définitivement ruinée.

D'autant plus ruinée qu'au désastre intérieur s'ajoutent les conséquences catastrophiques des actes commis durant cette crise et qui restent comme la marque objective et indélébile de cet état quasi frénétique. Si cette passion soudaine, violente, dévastatrice, exprimée dans le texte par la multiplication des métaphores empruntées aux forces naturelles- tempête, orage, foudre, feu, volcan - apparaît comme l'un des ressorts essentiels de Marie Stuart, elle est loin d'être l'apanage de cette seule personnalité et se présente au contraire comme une structure récurrente de nombreux personnages zweigiens. Car qu'il s'agisse de la narratrice de Vingt quatre heures de la vie d'une femme ou de celui de Amok, par exemple, la passion, chez Zweig, est toujours destructrice. Arrachant le sujet à son existence ordinaire, elle le plonge dans un état d'éréthisme où il ne se reconnaît plus et du fond duquel, balayant tous les gages d'une vie ordonnée, il détruit dans une sorte de fureur extatique tout ce qu'il a patiemment élaboré au cours de son existence. Généralisant le phénomène, tant il est convaincu de sa pertinence psychologique, Zweig écrit en effet :

Il y a des femmes qui dépensent en une seule passion toute leur réserve d'amour [...] En un seul élan ces femmes, véritables génies de l'autodestruction, se jettent dans les profondeurs de la passion d'où il n'y a plus ni retour, ni salut. De cette sorte d'amour [...] Marie Stuart restera un exemple parfait, elle [...] qui s'y adonna jusqu'au bout, jusqu'à l'annihilation totale de son $\mathrm{moi}^{27}$. 


\section{Les forces obscures de la passion} émergeant brutalement dans le champ de conscience du sujet, le dépossèdent de luimême, paralysent sa volonté et le privent de sa liberté. Ce caractère aliénant de la passion recouvre, chez les personnages zweigiens en général et chez Marie Stuart en particulier, deux aspects distincts mais complémentaires. En effet la passion est d'abord aliénante dans le sens où, s'emparant d'un sujet, elle l'arrache à sa vie ordinaire et l'assujettit corps et âme à un autre être pour l'amour duquel il sacrifie tout ce qui constituait jusqu'alors l'intérêt de sa vie.

列 ainsi que Marie Stuart, soumise à ce que Zweig nomme «l'action hypnotique ${ }^{28}$ » de Bothwell sur elle, abandonne sa propre volition pour ne plus agir qu'en fonction de celle de son amant ; devenue l'esclave de cet homme auquel elle ne peut résister, privée de tout discernement, c'est sous son emprise qu'elle accepte l'idée du meurtre de Darnley et qu'elle se fait la complice de son assassinat.

Mais la passion est aussi aliénante- et c'est cet aspect qui est surtout caractéristique des personnages zweigiens- en ce sens que le sujet est contraint par la force même de ses affects. C'est l'exaltation de ses sentiments qui le réduit et l'enchaîne. Apparaissant comme une force jaillie des profondeurs de l'être, elle plie le sujet à sa loi, égare sa raison, abolit sa volonté ; puissance intérieure jusque-là ignorée elle s'empare de la personne et l'entraîne à commettre les actes les plus révoltants ou les plus absurdes. La puissance de ce déterminisme psychologique est attestée à maintes reprises par le comportement de Marie Stuart, comme par celui de nombreux autres personnages et se trouve résumée dans ces lignes :

Marie Stuart n'est pas née pour la tranquillité et le bonheur. Sans cesse la pousse une force intérieure. Il apparaît une fois de plus que ce ne sont pas les événements et les hasards du monde extérieur qui impriment sa forme et son sens à une destinée. Ce sont toujours les lois innées propres à l'individu qui créent ou détruisent une vie ${ }^{29}$.

De sorte qu'ainsi menés par cette force intérieure irrésistible, les personnages zweigiens apparaissent comme de véritables possédés, la passion qui les habite et contre laquelle ils luttent en vain évoquant une puissance occulte, un démon, semblable à celui qu'affrontent durant leur vie Kleist, Hölderlin et Nietzsche auxquels Zweig consacre un essai précisément intitulé Le combat avec le Démon. C'est que passion et folie ont à voir l'une avec l'autre dans ce jeu de forces obscures car, en divisant le moi du sujet en deux entités opposées- d'un côté le moi "possédé ", obéissant à la logique passionnelle, de l'autre, le moi commun, façonné par les lois sociales et morales et guidé par les intérêts supérieurs du sujet- la passion détruit sa cohésion entrainant l'individu aux limites de la déraison ou du suicide.

D'autant que les prérogatives du moi social sont nécessairement contrariées par les forces de la passion, celle-ci balayant les structures supérieures de l'être dans la mesure où le personnage lui sacrifie tous les principes qui régissaient jusqu'alors sa vie. Ce sacrifice est une constante du comportement des personnages zweigiens asservis à la passion dont l'évocation donne lieu à des passages narratifs d'une très grande intensité. Tous abandonnent en effet, toujours dans un instant de véhémence d'où réflexion et raisonnement sont abolis, les préceptes qui les gouvernaient, les règles intangibles sur lesquelles ils s'appuyaient. Qu'il s'agisse de la narratrice de Vingt- quatre heures de la vie 
d'une femme avouant qu'elle aurait bien été " prête à jeter d'un seul coup dans l'abîme toutes les réserves d'une vie bien administrée ${ }^{30}$ » et à « sacrifier (à un homme rencontré par hasard) son nom (et) celui de ces enfants, son argent et son honneur ${ }^{31} »$, ou de Marie Stuart dont Zweig affirme que son amour pour Bothwell lui fait « oublier tout: morale, loi, honneur, couronne, pays ${ }^{32}$ ", c'est toujours le même bouleversement de l'être qui, pour quelques moments d'exaltation et de vie éperdue, renonce aux principes sociaux, moraux et culturels supérieurs.

\section{Fascination de Zweig pour la passion}

Toutefois la crise passionnelle en dépit de son caractère dévastateur, découvre au sujet une vision nouvelle de la vie et de ses profondeurs ; transformé intérieurement par le tumulte de ses sentiments et la confrontation à des forces qui le dépassent, celui-ci perçoit en effet l'intensité insoupçonnée de la vie tout en découvrant une vérité de luimême jusqu'alors méconnue. Si bien que cette crise douloureuse apparaît dès lors, et paradoxalement, comme une expérience susceptible d'approfondir le sens de la vie et de l'être, «c'est toujours la passion, dit Zweig, qui dévoile à une femme son caractère, c'est toujours dans l'amour et dans la douleur qu'elle atteint sa véritable mesure ${ }^{33}$. Ouvrant sur le mystère de la psyché, manifestant l'activité des forces inconscientes, menaçant l'intégrité du moi tout en révélant l'être à lui-même, la passion exerce une véritable fascination sur Zweig qui, œuvre après œuvre, s'attache à cerner les mécanismes secrets qui déterminent les sentiments humains, tant il est vrai, comme il le dit si bien à propos de Dostoïevski que, sur lui aussi, « la profondeur du cœur humain exerce une attraction magique ${ }^{34} »$.

Au reste, c'est cette fascination pour la passion qui l'amène à donner une interprétation finalement très personnelle du personnage de Marie Stuart en faisant de son amour passionné pour Bothwell le pivot de sa tragique destinée alors que l'importance de cette relation peut tout aussi bien être minimisée, et son effet sur le cours des événements envisagé tout à fait différemment. C'est ce que démontre, par exemple, Paule Henri-Bordeaux, une historienne de l'Entre-deux-guerres, pour qui Bothwell est avant tout un jouet entre les mains du Comte de Morray, et pour qui le drame de Marie Stuart résulte d'abord de la rivalité de celui-ci avec Bothwell pour la couronne d'Écosse.

Ainsi, figure du monde finissant de la Renaissance et de l'humanisme dont elle incarne les valeurs et l'esprit, jouet de forces qui la dépassent, victime de la violence de sa passion, c'est donc bien, nous semble-t-il, comme un véritable personnage zweigien qu'apparaît Marie Stuart dans le récit. Si cette dimension semble bien attestée par la proximité de la reine d'Écosse avec d'autres héros ou héroïnes de l'œuvre de Zweig, il reste dès lors à définir le statut même de ce personnage historique tellement façonné par la thématique de l'auteur, de même que celui, entre Histoire et fiction, de la biographie elle-même et de voir enfin comment Zweig parvient à dépasser les contradictions entre fiction narrative et vérité historique. 


\section{Entre Histoire et fiction : statut de la biographie chez Zweig}

\section{Personnalité historique ou personnage de littérature ?} truisme puisque c'est bien les épisodes de la vie mouvementée de la dernière reine d'Écosse qu'expose, avec une rigoureuse exactitude documentaire, le récit de Zweig. Les trois mariages successifs de la souveraine - dont l'un avec l'héritier du trône de France - ses veuvages - dont l'un consécutif à un assassinat - sa longue captivité en Angleterre et, enfin, son exécution sont en effet les étapes historiques avérées qui constituent la trame narrative de la biographie. Cependant c'est là à peu près tout ce que cette Marie Stuart historique a de commun avec le personnage dont Zweig nous dévoile les méandres de l'âme et les conflits intérieurs. En effet, parce que la Marie Stuart de l'œuvre illustre les grands motifs de la thématique de l'auteur, parce qu'elle est un véritable personnage zweigien, elle se présente déjà comme une entité d'ordre littéraire. De plus, parce que Zweig ne peut faire totalement abstraction, malgré sa volonté d'aborder son sujet avec le plus d'impartialité possible, de la vision que trois siècles de commentaires et d'interprétations ont léguée de la reine d'Écosse, il reste tributaire de l'aura quasi mythique qui entoure Marie Stuart.

est pourquoi commençant par évoquer son aspect physique en se fondant sur les tableaux des peintres de l'époque mais en n'ignorant pas les évocations faites par de très nombreux poètes, Zweig trace le portrait d'une jeune femme dont la beauté n'est pas sans évoquer les topoï des représentations physiques des héroïnes de légende. En effet, dotée "d'une grâce particulière ", Marie Stuart est très belle avec " une peau étincelante de blancheur, une chevelure blond cendré, luxuriante, des mains fines et blanches, un buste élancé et souple $»^{35}$, esthétique qui fait songer à la reine Guenièvre ou à Iseult la Blonde par exemple, et qui contribue à situer le personnage dans le domaine du mythe. Situation que Zweig ne cesse de conforter en insistant notamment, comme nous l'avons vu, sur le fait que Marie Stuart s'impose davantage, aux yeux de la postérité, comme une figure légendaire cependant qu'Elisabeth se fixe, comme dans ses portraits, en personnage historique hiératique.

romantique est pour Zweig, comme nous l'avons vu précédemment, un des qualificatifs essentiels définissant la personnalité de Marie Stuart, romanesque en est un autre non moins fondamental en ce qu'il exprime la représentation que l'auteur se fait de son personnage. Au reste il semble qu'il y ait parfois un glissement sémantique d'un terme à l'autre, Zweig employant le mot romantique dans le sens de romanesque.

Quoi qu'il en soit, cette dimension romanesque de Marie Stuart est plusieurs fois affirmée tant pour évoquer son absence de réalisme face aux circonstances difficiles que pour dépeindre le côté sentimental de sa personne et de certains de ses comportements et, surtout, pour définir cette tendance particulière qui l'incline à vivre les événements de l'existence comme de véritables aventures de sorte que, pleine d'ardeur et d'impétuosité, elle s'engage souvent dans des situations hasardeuses quant à leurs conséquences mais dans lesquelles, se livrant totalement, elle goûte la plénitude de l'instant présent.

D'ailleurs, c'est la vie entière de Marie Stuart qui, dans la vision de Zweig, donne lieu à une comparaison avec la littérature. Tout au long de son récit la vie de la reine d'Écosse 
est en effet envisagée comme une œuvre littéraire et, elle-même, comme le personnage littéraire de sa vie. Placée dès le début, ainsi que nous l'avons remarqué, dans la lignée des héroïnes tragiques grecques et shakespeariennes, sa vie est explicitement comparée à une tragédie dont les différents actes et scènes sont constitués par les grandes étapes de son existence, la « naissance de son enfant, écrit par exemple Zweig, représente dans la tragédie de Marie Stuart la fin du premier acte, lequel n'est que la préparation de l'action ${ }^{36}$ ». De même, c'est une longue « ballade tragique et passionnée ${ }^{37}$ " qu'évoque le narrateur lorsqu'il considère la vie de son personnage dans son ensemble, comparaison d'autant plus signifiante quand on se rappelle qu'issue de l'héritage lyrique médiéval, la ballade était réapparue au XIXe siècle sous la forme d'un poème populaire développant un thème légendaire. «Maintenant commence la strophe la plus sombre de la ballade de Marie Stuart ${ }^{38}$ " note-t-il encore lorsque celle-ci s'engage dans la voie du meurtre de Darnley.

Enfin, s'il était encore nécessaire d'illustrer cette correspondance entre la vie de la reine d'Écosse et la littérature afin d'établir l'indubitable caractère littéraire que revêt ce personnage historique dans l'œuvre de Zweig, on pourrait encore remarquer que c'est au poème héroïque, qui mêle légende et histoire, qu'il recourt pour évoquer la mise en scène de la mort de Marie Stuart, "Chaque détail, écrit-il ainsi, à sa place comme une strophe touchante ou puissamment émouvante, dans le poème héroïque d'une mort exemplaire ${ }^{39}$ ».

Il resterait surtout à considérer le lien particulier que Zweig établit entre la vie de Marie Stuart et l'action des drames shakespeariens. Car non seulement il évoque Hamlet et Richard III et établit un parallèle entre l'état d'esprit troublé de Lady Macbeth et celui de la reine d'Écosse lors de l'assassinat de Darnley, mais il va plus loin en suggérant un lien entre l'inspiration de Shakespeare et la réalité historique des événements de la vie de Marie Stuart. Le dramaturge ayant pu selon lui fondre (consciemment ou non) des éléments du drame historique dans le récit fourni par la chronique d'Hollinshed. Ne pouvant prouver cette hypothèse, il n'en conclut pas moins que pour saisir toute la profondeur du drame de Marie Stuart, il faut avoir compris celui qui ronge Lady Macbeth, réaffirmant ainsi de façon implicite la nature fondamentalement littéraire de son personnage.

\section{Une fiction tissée dans la trame lacunaire de l'Histoire}

57 S'il a été ainsi possible à Zweig d'aborder littérairement le personnage de Marie Stuart c'est certes que la personnalité et la vie mouvementée de la reine d'Écosse s'y prêtaient, mais c'est surtout que l'histoire incertaine, contradictoire et lacunaire laissait planer autour de cette femme énigmatique de larges zones d'ombres dans lesquelles la fiction pouvait s'insérer presque naturellement. Car comme il est courant en histoire, il y a beaucoup d'inconnu dans le règne de Marie Stuart, mais contrairement à ce qu'il advient généralement, cet inconnu ne résulte pas ici de l'absence de matériau mais au contraire, ainsi que Zweig le remarque dès la préface, de la surabondance des documents, de la profusion des témoignages qui se contredisent les uns les autres, du nombre impressionnant de versions opposées d'un même événement. Toutes les étapes de la crise vécue par Marie Stuart entre vingt-trois et vingt-cinq ans étant en effet rapportées de façon fort différente selon l'origine de ceux qui les relatent. Et selon que 
l'on suit tel ou tel commentateur l'interprétation des faits, et plus encore de leurs causes, peut varier d'une affirmation à son contraire.

Ainsi de la date à laquelle on fait débuter la liaison entre Marie Stuart et Bothwell on peut affirmer que le meurtre de Darnley ressortit à un crime passionnel dans lequel la reine est pleinement impliquée ou, au contraire, contester cette hypothèse. Car si l'amour fou de Marie pour Bothwell date, comme l'avance Zweig, d'avant octobre 1566 on conçoit, et c'est la thèse de l'auteur, que celle-ci subjuguée par une passion de plusieurs mois, se soit rendue complice d'un crime qui la délivrait des entraves du mariage et lui procurait la liberté de vivre ouvertement son amour illégitime. Mais cette date est loin d'être avérée et certains historiens, comme Michel Duchein, la contestent fermement. De sorte que l'on saisit alors comment l'incertitude historique offre à Zweig une ouverture lui permettant d'insérer sa vision romanesque dans la trame des événements en retenant des deux visions celle qui s'harmonise avec sa conception d'un personnage assujetti à la passion, un amour vieux de seulement deux mois ne pouvant être crédible comme mobile d'un acte aussi grave.

De même un doute plane sur les motivations qui poussèrent Marie Stuart à rejoindre son mari malade à Glasgow et à le ramener, souffrant et peut-être encore contagieux, à Édimbourg où il périt quelques jours plus tard dans l'explosion de la demeure où il s'était retiré. Là encore l'histoire laisse un blanc dans lequel chaque commentateur glisse son interprétation. Ainsi pour Michel Duchein, Marie Stuart, avertie d'un complot tramé contre son fils (le futur Jacques $1^{\text {er }} d^{\prime}$ 'Angleterre) par Darnley et son père le Comte de Lennox, serait allée chercher son mari afin de neutraliser ses perfides manœuvres. Mais pour Zweig, il est tout à fait évident que Marie s'est rendue à Glasgow chercher son époux, poussée par la volonté irrésistible de Bothwell. Se fondant sur l'examen de la fameuse lettre écrite dans la nuit passée au chevet de Darnley appartenant à cet ensemble désigné sous la dénomination de « lettres de la cassette », il démontre dans une analyse psychologique digne de Dostoïevski -le maître du genre selon lui- les mécanismes profonds de son infamie commise malgré elle sous l'emprise des forces obscures de la passion.

Avec ce corpus d'écrits très controversés attribués à la reine d'Écosse, les lacunes de l'histoire se révèlent en effet dans toute leur ampleur. Car ces lettres, pièces décisives dans l'établissement de sa culpabilité dans le meurtre de Darnley, n'ont jamais revêtu un caractère absolument incontestable puisque les exemplaires qui ont circulé, même du vivant de la reine, n'étaient pour la plupart que des copies voire des copies de copies, et qu'ils ont fini par disparaitre tout à fait peu de temps après sa mort, probablement détruits par Jacques $1^{\text {er }}$ désireux de mettre un terme au déshonneur de sa mère. Dans ces conditions, où seule une expertise graphologique à jamais impossible pourrait trancher, trois positions envers ces documents sont dès lors possibles.

61 La première consiste à considérer ces écrits comme absolument faux, rédigés purement et simplement par les adversaires politiques les plus farouches de Marie Stuart, en tête desquels se détachent le chancelier d'État Maitland de Lethington et le poète humaniste George Buchanan qui publia par la suite des pamphlets haineux dirigés contre la reine. C'est là le parti des défenseurs de Marie Stuart, notamment au début du XIXième siècle où les malheurs et les charges pesant sur la reine d'Écosse furent repris et exploités à des fins politiques en France par le parti légitimiste. C'est encore le point de vue adopté avant la guerre par l'historienne de droite Paule Henry- Bordeaux. La seconde attitude est d'envisager l'ensemble des documents comme partiellement faux, c'est-à-dire de 
considérer les lettres notamment la plus importante, que l'on nomme « la longue lettre de Glasgow ", comme une falsification d'un écrit authentique ; c'est la thèse défendue par Michel Duchein lorsqu'il écrit : «Quant à la longue lettre de Glasgow, elle serait une sorte de patchwork où seraient mêlés des éléments d'une authentique lettre de Marie à des passages apocryphes destinés à l'incriminer ${ }^{40}$ ». A cette interprétation médiane, partagée par la plupart des historiens modernes, s'oppose enfin la troisième posture qui consiste à tenir les missives pour absolument authentiques. C'est bien entendu celle qui rallie tous les adversaires de Marie Stuart à commencer par le Comte de Lennox, père de Darnley, ainsi que la grande majorité des Protestants, et les Anglais en général. Mais c'est aussi celle qu'adopte Zweig en développant ses idées sur la passion et en démontrant par là l'irresponsabilité de Marie Stuart dans une conduite qui lui a été dictée par la force irrésistible de ses sentiments. De sorte que le paradoxe qui le conduit à reprendre à son compte les accusations portées par les pires ennemis de la reine n'est qu'apparent puisqu'il ne s'agit pas pour lui de la défendre en prouvant son innocence, mais bien de montrer la puissance des forces obscures qui la gouvernaient.

Ainsi dans son commentaire de la lettre de Glasgow qui révèle son enthousiasme à comprendre les mécanismes profonds du psychisme, le romancier viennois se livre à une analyse que n'aurait pas désavouée son illustre compatriote Freud. Comblant les hiatus de l'histoire par sa vision romanesque d'une Marie Stuart esclave de sa passion, il interprète en effet les incohérences de la lettre, que Michel Duchein attribue au caractère composite $\mathrm{du}$ texte, comme le signe incontestable du bouleversement psychique du personnage. Observant ainsi que «la lettre n'est pas écrite d'une façon claire et lucide », il en déduit qu'il s'agit « d'un document psychologique précieux (...). Car, dit-il, ce n'est pas (ici) l'être conscient qui parle, mais le moi intérieur, dans une sorte d'hallucination provoquée par la fatigue et la fièvre, ici parle le subconscient ${ }^{41}$ ".

Saisissant ainsi les contradictions et les incertitudes laissées par l'histoire officielle autour des événements emmêlés de la vie de Marie Stuart, Zweig a senti la possibilité de faire de la reine d'Écosse une illustration de sa vision romanesque de la passion entraînant un être profondément moral et humain dans la voie du crime.

\section{Fiction narrative, vérité historique : l'art comme dépassement des contraires}

\section{L'empathie, élément de la création chez Zweig}

Écrivant donc entre les lignes de l'Histoire officielle, Zweig pose dans son ouvrage sur Marie Stuart la délicate question des rapports entre fiction et vérité historique ainsi que celle, non moins complexe, du statut de la biographie.

Si l'on admet comme une caractéristique essentielle du roman historique l'exposition d'une problématique de l'histoire (ce que l'on désigne couramment par le terme de problématisation), on peut sans doute considérer la démonstration plus ou moins explicite de l'incapacité de Marie Stuart, et des puissances catholiques qui la soutenaient, à s'opposer au progrès de la Réforme et à contenir la montée en puissance de l'Angleterre, comme un élément allant dans ce sens.

De même, il serait possible de voir dans l'affirmation, plusieurs fois réitérée, de la légitimité de Marie Stuart à régner sur le trône d'Écosse, et dans sa conviction à considérer sa captivité en Angleterre comme une violation absolue du droit et de la 
justice, une dénonciation implicite du régime nazi et une condamnation de l'iniquité d'un pouvoir bafouant les droits humains les plus fondamentaux. A l'appui de cette interprétation on pourrait relever l'emploi, anachronique pour l'époque, des termes «dictateur » et «dictature » qualifiant à plusieurs reprises Bothwell et ses menées politiques après le meurtre de Darnley. Quoi qu'il en soit, même si cette lecture de l'histoire passée à l'aune des événements présents est pertinente, il n'en reste pas moins que l'intérêt de l'œuvre de Zweig se situe sur un tout autre plan et que l'utilisation de l'histoire dans une perspective didactique est largement surpassée par une stratégie narrative plus ouvertement tournée vers le romanesque pur.

Déjà auteur de plusieurs biographies notamment de celle de Marie-Antoinette en 1933, Zweig avec ce genre littéraire, semble trouver la forme adéquate à l'expression de quelques unes des ses convictions les plus intimes. En premier lieu, celle-ci lui offre l'occasion d'exprimer son intérêt pour les moments exceptionnels de l'histoire, ceux qu'il nomme "ces heures étoilées» où se condense en quelques événements extrêmement denses ce qui d'ordinaire se déroule sur des durées infiniment plus longues, ces moments qui «décident de tout et déterminent le destin d'un peuple, d'une génération, ou même de l'humanité entière ${ }^{42} »$. Et c'est un tel moment qu'il voit par exemple s'accomplir dans la transition historique qui s'opère dans le duel entre Marie Stuart et Elisabeth $1^{\text {ère }}$ où la marche de l'histoire semble soudain s'accélérer. Mais la biographie le captive surtout en cela qu'elle lui permet de mettre en lumière, chez une personnalité réelle et non plus fictive comme dans ses romans et nouvelles, l'effet dynamique des forces qui, dans certaines circonstances, contraignent un sujet à agir contre sa volonté jusqu'à détruire sa propre vie poussé par des mécanismes émanant des zones profondes de l'être. C'est ce qu'illustre avec éclat son évocation de Marie Stuart voyant son destin lui échapper sous la pression des forces qui déterminent son aveuglante passion.

De sorte que la question se pose alors de savoir ce qui amène Zweig à mettre en scène dans sa création des personnages dont la destinée malheureuse résulte d'actes si singuliers. Même si, comme le mentionne Serge Niemetz dans sa biographie "l'intérêt de Zweig pour l'arrière plan psychologique des personnalités dont il traite dans ses essais ou des personnages fictifs de ses nouvelles s'inscrit dans la tradition viennoise " de son époque et « rappelle les analyses d'Arthur Schnitzler " ${ }^{43}$, il n'en reste pas moins que cette tendance dérive d'abord de sa curiosité d'artiste à comprendre par la "participation affective et l'identification ${ }^{44}$ " les mouvements profonds de l'âme humaine. Et c'est indubitablement la clé de sa propre inspiration qu'il livre au travers des propos du narrateur de Amok lorsque celui-ci avoue «Les énigmes psychologiques ont sur moi une sorte de pouvoir inquiétant; je brûle de tout mon être de découvrir le rapport des choses, et des individus singuliers peuvent par leur seule présence déchaîner en moi une passion de savoir qui n'est guère moins vive que le désir passionné de posséder une femme ${ }^{45}$ ». Et cette passion à comprendre les êtres, fondée sur une empathie qui lui permet de s'identifier à un personnage, notamment par le moment de crise qui révèle le sujet à lui-même, apparaît comme la caractéristique la plus originale de son génie créateur. En outre c'est cette empathie, cette " compréhension par la sympathie ${ }^{46}$ " selon l'expression de Romain Rolland, qui lui permet, lorsqu'il s'attache à un personnage historique comme Marie Stuart, de pallier l'ambiguïté des documents et de combler les lacunes de l'histoire. 
69 Percevant de manière empathique c'est-à-dire saisissant le monde intérieur de son personnage comme s'il était lui-même ce personnage, il pourvoit en effet aux insuffisances et aux imprécisions incontournables des données historiques par le pouvoir de l'imagination; le récit historiographique étant nécessairement lacunaire dans la mesure même où il ne restitue d'un événement ou d'un personnage qu'une vision abstraite et désincarnée. Si bien que seule la fiction créée par l'imagination du romancier peut suppléer la réalité à jamais perdue d'un épisode et animer d'un souffle lyrique, poétique ou tragique ce qui n'est sinon qu'accumulation d'observations et de remarques érudites. C'est très exactement ce que fait Zweig qui, tout en respectant rigoureusement les éléments historiques, recrée une atmosphère, une épaisseur, qui redonnent vie aux événements de l'existence de Marie Stuart. Au reste, certaines modalisations du récit prouvent à quel point le romancier est conscient de ces procédés; ainsi peut-on lire à propos d'une scène entre Marie et Bothwell ce commentaire qui atteste la position non historique du narrateur, «Probablement - on croit voir la chose - (c'est nous qui soulignons) se permet-elle de temps en temps avec Bothwell une de ces innocentes familiarités, une de ces coquetteries féminines qui lui ont déjà été si funestes avec Chastelard et Riccio ${ }^{47}$ ? ». De sorte qu'en dépit de toutes les affirmations d'impartialité fournies par l'auteur qui se définit tour à tour comme « un historien impartial ${ }^{48} »$, « un chercheur impartial ${ }^{49} »$, et même tout à fait paradoxalement, comme un "artiste impartial ${ }^{50}$ ", son " essai » sur Marie Stuart s'apparente bien à un roman historique, d'autant qu'en menant son récit comme une véritable enquête sur une série de complots meurtriers, Zweig restitue aux événements historiquement avérés et parfaitement connus une dimension d'aventure propre à la fiction romanesque.

70 Ce faisant, il ne vise pas à l'exactitude historique mais bien plutôt à donner âme et vie à une personnalité qui le fascine et qui fascine aussi le public parce qu'elle s'inscrit depuis plusieurs siècles dans l'imaginaire collectif. Ce qu'il recherche avec la biographie c'est plus un impact affectif sur le lecteur qu'une reconstitution de la vérité historique $\mathrm{du}$ personnage, ce qui explique la fréquence d'images saisissantes et de scènes fortes visant à émouvoir, tout au moins à frapper l'imagination du lecteur. Ainsi en témoigne, parmi de nombreux exemples, celle de la nuit de Glasgow montrant une Marie Stuart hallucinée, écartelée entre sa conscience et sa passion, écrivant à Bothwell la fameuse «longue lettre de la cassette». Une telle scène, portant à son comble l'effet dramatique, n'aurait probablement pas été reniée par Walter Scott lui-même, tant elle relève plus du genre romanesque que de l'écrit historique objectif. C'est donc en fusionnant, au sein du récit, la fiction narrative et les éléments documentaires attestés par l'histoire afin de créer une figure vivante, incarnée, et surtout inoubliable, que Zweig élève le genre singulier de la biographie historique à son expression la plus achevée.

71 Séduit par la figure troublante de la dernière reine d'Écosse, fasciné par cette personnalité sacrifiant, au terme d'une lutte intérieure destructrice, ses prérogatives royales aux exigences de sa passion, frappé enfin par sa situation au carrefour de deux époques où s'affrontent deux conceptions du monde, Zweig, explorateur inlassable et passionné des profondeurs du cœur humain, a tenté de percer ce qu'un biographe contemporain a nommé "l'Énigme Marie Stuart ${ }^{51}$ ». Mais loin d'une enquête qui détaillerait les éléments objectifs d'un règne dont le rayonnement purement historique ne saurait égaler celui de son irréductible rivale, la Grande Elisabeth, c'est un récit 
romanesque d'une grande intensité dramatique que compose Zweig, où Marie Stuart triomphe définitivement sur le plan du mythe et non sur celui de l'Histoire.

\section{BIBLIOGRAPHIE}

\section{Les citations de Zweig sont extraites des éditions suivantes :}

Marie Stuart,(Maria Stuart, Insel Verlag, Leipzig, 1935), traduction d'Alzir Hella, Edition Bernard Grasset, Collection le Livre de Poche Historique, Paris, 1958.

Amok, (Amok, Novellen einer Leidenschaft), traduction par Alzir Hella et Olivier Bournac revue par Brigitte Vergne-Cain et Gérard Rudent, Préface de Romain Rolland, le Livre de Poche, Paris, 1991.

Le Monde d'Hier - Souvenirs d'un Européen, (Die Welt von Gesten - Erinnerungen eines Europäers), traduction nouvelle de Serge Niémetz, Belford, le Livre de Poche, Paris, 1993.

Marie-Antoinette, traduction Alzir Hella, les Cahiers Rouges, Grasset, Paris, 2006.

Euvres, Stefan Zweig «Classiques Modernes ", la Pochothèque, le Livre de Poche, Librairie Générale de France, Paris, 1991, 1995, 1996.

Trois Maîtres, (Drei Meister), Stefan Zweig III « Classiques Modernes », la Pochothèque, le Livre de Poche, Librairie Générale de France, Paris, 1991, 1995, 1996.

Vingt-quatre heures de la vie d'une femme,(Vierundzwanzig Stunden aus dem Lebeneiner Frau), traduction et introduction par Olivier Bournac et Alzir Hella - Révision de Brigitte Vergne-Cain et Gérard Rudent, le Livre de Poche, Paris 1980.

\section{Autres ouvrages consultés}

CADENE, Nicole. Marie Stuart en France 1820-1890, thèse de doctorat, Université de Nice.

Correspondance Sigmund Freud-Stefan Zweig, (Stefan Zweig - Briefwechsel mit Sigmund Freud) traduction Didier Plassard et Gisella Hauer, Rivages poche, Petite Bibliothèque, Paris 1995.

DUCHEIN, Michel. Marie Stuart la Femme et le mythe, Fayard, Paris, 1987.

FRYE, Northrop. L'Ecriture profane.Essai sur la structure du romanesque, traduction de Cornélius Crowley, Bibliothèque critique, Circé, Paris, 1998.

HENRY-BORDEAUX, Paule. Marie Stuart, Reine de France et d'Ecosse (I), les Prisons et la mort (II), Plan, Paris, 1938.

La Passion, Nouvelle Revue de Psychanalyse, Numéro 21 - Printemps 1980, Gallimard.

Le Roman historique. Récit et Histoire, sous la direction de Dominique Peyrache- Leborgne et Daniel Couégnas, Collection « Horizons comparatistes », Université de Nantes, Editions Pleins Feux, Nantes, 2000.

LUKACS, Georges. Le Roman historique, préface de Claude-Edmonde Magny, $n^{\circ} 2311$ Petite Bibliothèque Payot, Paris, 1965. 
MAIGRON, Louis. Le Roman historique à l'Epoque Romantique Essai sur l'influence de Walter Scott, Librairie Ancienne H. Champion, Editeur, Paris, 1912.

MOLINO, Jean : Qu'est-ce que le roman historique? In Revue d'Histoire Littéraire de la France, MarsJuin 1975.

MUHLSTEIN, Anka. Elisabeth d'Angleterre et Marie Stuart ou les périls du mariage, Albin Michel, Paris, 2004.

NIEMETZ, Serge. Stefan ZWEIG le Voyageur et ses mondes, Biographie, Belfond, le Livre de Poche, Paris, 1996.

SCHILLER, Friedrich. Marie Stuart, traduction et post face de Sylvain Fort, l'Arche, Paris, 1998.

SCOTT, Walter. L'Abbé, CEuvres de Walter Scott, Tome X traduction Defauconpret, Furne, Jouvet et Cie. Garnier Frères libraires-éditeurs.

\section{NOTES}

1. Marie Stuart, Insel Verlag, Liepzig, 1935, Le Livre de poche, Paris, 1958.

2. Marie Stuart, p. 18.

3. Marie Stuart, p. 243.

4. Marie Stuart, p. 96.

5. Marie Stuart, p. 445.

6. Marie Stuart, p. 57.

7. Marie Stuart, p. 73.

8. Marie Stuart, p. 61.

9. Marie Stuart, p. 97.

10. Marie Stuart, p. 436.

11. Marie Stuart, p. 89-90.

12. Friedrich Schiller, Marie Stuart, ed. Bilingue par H. Loiseau, ed. Aubier-Montaigne, Paris, 1964.

13. Marie Stuart, p. 198.

14. Marie Stuart, p. 89.

15. Marie Stuart, p. 68.

16. Marie Stuart, p. 79.

17. Marie Stuart, p. 79.

18. Marie Stuart, p. 32.

19. Serge Niemetz, Stefan Zweig, Le Voyageur et ses mondes, Belfond, le Livre de Poche, Paris, 1996, p. 96.

20. Serge Niemetz, Stefan Zweig, Le Voyageur et ses mondes, p.49.

21. Marie Stuart, pp. 34-156-63.

22. Marie Stuart, p. 63.

23. Marie Stuart, pp. 114 et 117.

24. Marie Stuart, p. 132.

25. Marie Stuart, p. 138.

26. L'amour d'Erika Ewald, la Pochotèque, Sfefan Zweig, II, p. 55.

27. Marie Stuart, p. 215.

28. Marie Stuart, p. 247.

29. Marie Stuart, p. 191.

30. Vingt-quatre heures de la vie d'une femme, Le Livre de Poche, 1980, p. 112.

31. Vingt-quatre heures de la vie d'une femme, p. 105. 
32. Marie Stuart, p. 193.

33. Marie Stuart, p.37.

34. Trois Maîtres, Dostoïevski, Stefan Zweig, La Pochotèque III, p. 172.

35. Marie Stuart, p. 36.

36. Marie Stuart, p. 192.

37. Marie Stuart, p. 316.

38. Marie Stuart, p. 243.

39. Marie Stuart, pp. 476-477.

40. Michel Duchein, Marie Stuart la Femme et le mythe, Fayard, Paris, 1987, p. 383.

41. Marie Stuart, pp. 250-251.

42. Les très riches heures de l'humanité - cité par Serge Niemetz dans Stefan Zweig, le Voyageur et ses mondes, p. 473.

43. Serge Niemetz, Stefan Zweig, Le Voyageur et ses mondes, p. 457.

44. Serge Niemetz, Stefan Zweig, Le Voyageur et ses mondes, p. 288.

45. Stefan Zweig, Amok, Stefan Zweig, La Pochotèque, p. 33.

46. Serge Niemetz, Stefan Zweig, Le Voyageur et ses mondes, p.524.

47. Marie Stuart, p. 224 (les tirets sont de Zweig).

48. Marie Stuart, p. 223.

49. Marie Stuart, p. 246-247.

50. Marie Stuart, Préface, p. XI.

51. Ian B. Cowan, The Enigma of Mary Stuart, 1971.

\section{RÉSUMÉS}

Partant du constat qu'aucune figure historique n'a, autant que Marie Stuart, suscité de débats et de controverses chez les historiens comme chez les romanciers, Stefan Zweig se propose, dans son ouvrage consacré à la dernière reine d'Ecosse, d'aborder cette personnalité auréolée de mystère avec l'impartialité du psychologue et la ferveur de l'artiste. Ce faisant, il livre une œuvre singulière à propos de laquelle on peut se demander si sa neutralité, garantie par son recul par rapport aux causes idéologiques et politiques de l'époque, n'est cependant pas subvertie par sa sensibilité même d'artiste, c'est-à-dire si sa reine d'Ecosse n'est pas un personnage fortement fictionnel, et si son « essai » biographique ne se rattache pas en fait au statut générique du roman historique.

\section{INDEX}

Mots-clés : biographie, genre, Histoire, humanisme, passion, personnage, reine, roman-fiction Index géographique : Angleterre, Ecosse 


\section{AUTEUR}

\section{FRÉDÉRICA ZEPHIR}

Chargée de cours de littérature comparée à l'Université de Nice Sophia-Antipolis. Rattachée au centre Interdisciplinaire Récits, Cultures, Psychanalyse, Langues et Société (CIRCPLES) de l'Université de Nice. 